\title{
Validation of diagnostic characteristics of needle based confocal laser endomicroscopy in differentiation of pancreatic cystic lesions
}

Authors

Institutions
Somashekar G. Krishna ${ }^{1,2}$, Benjamin Swanson ${ }^{3}$, Phil A. Hart ${ }^{2}$, Samer El-Dika ${ }^{1,2}$, Jon P. Walker ${ }^{1}$, Sean T. McCarthy ${ }^{1,2}$, Ahmad Malli ${ }^{1}$, Zarine K. Shah ${ }^{4}$, Darwin L. Conwell ${ }^{2}$

Institutions are listed at the end of article. submitted 25. May 2016 accepted after revision 19. August 2016

\section{Bibliography}

Dol http://dx.doi.org/

$10.1055 / \mathrm{s}-0042-116491$

Published online: 30.9.2016

Endoscopy International Open 2016; 04: E1124-E1135

(c) Georg Thieme Verlag KG

Stuttgart · New York

E-ISSN 2196-9736

\section{Corresponding author}

Somashekar G. Krishna, MD MPH

395 W. 12th Avenue

Suite 262

Division of Gastroenterology,

Hepatology and Nutrition

Columbus

Ohio

USA

Fax: +1-614-293-8518

sgkrishna@gmail.com

\section{License terms}

()(1) $\Theta \circledast$
Background and aims: Endoscopic ultrasound (EUS)-guided needle-based Confocal Laser Endomicroscopy (nCLE) characteristics of pancreatic cystic lesions (PCLs) have been identified in studies where the gold standard surgical histopathology was available in a minority of patients. There are diverging reports of interobserver agreement (IOA) and paucity of intraobserver reliability (IOR). Thus, we sought to validate current EUSnCLE criteria of PCLs in a larger consecutive series of surgical patients.

Methods: A retrospective analysis of patients who underwent EUS-nCLE at a single center was performed. For calculation of IOA (Fleiss' kappa) and IOR (Cohen's kappa), blinded nCLE-naïve observers $(n=6)$ reviewed $n C L E$ videos of PCLs in two phases separated by a 2-week washout period. Results: EUS-nCLE was performed in 49 subjects, and a definitive diagnosis was available in 26 patients. The overall sensitivity, specificity, and accuracy for diagnosing a mucinous PCL were $94 \%$, $82 \%$, and $89 \%$, respectively. The IOA for differen-

\section{Introduction \\ $\nabla$}

Approximately $2.5-10 \%$ of cross-sectional imaging studies report at least one incidental pancreatic cystic lesion (PCL) $[1,2]$. While pre-surgical differentiation of PCLs is challenging, appropriate surveillance of pancreatic cysts with malignant potential and resection of high risk lesions are important opportunities for prevention of pancreatic adenocarcinoma. To evaluate cysts, a combination of clinical history, imaging and endoscopic ultrasound (EUS) features, cytology, and cyst fluid carcinoembryonic antigen (CEA) is used to identify mucinous cysts [3]. This approach remains inadequate and may result in unnecessary surgery [4]. Although operative mortality associated with pancreatic surgery has decreased, considerable morbidities still occur in approximately one-third of patients $[4,5]$. tiating mucinous vs. non-mucinous PCL was "substantial” ( $\mathrm{K}=0.67,95 \% \mathrm{CI} 0.57,0.77)$. The mean ( \pm standard deviation) IOR was "substantial" ( $\mathrm{K}=$ $0.78 \pm 0.13$ ) for diagnosing mucinous PCLs. Both the IOAs and mean IORs were "substantial" for detection of known nCLE image patterns of papillae/ epithelial bands of mucinous PCLs (IOA $\mathrm{K}=0.63$; IOR $\mathrm{K}=0.76 \pm 0.11)$, bright particles on a dark background of pseudocysts $(\mathrm{IOA} \mathrm{K}=0.71$; IOR $\mathrm{K}=$ $0.78 \pm 0.12$ ), and fern-pattern or superficial vascular network of serous cystadenomas $(\mathrm{IOA} \mathrm{K}=0.62$; IOR $\mathrm{K}=0.68 \pm 0.20)$. Three $(6.1 \%$ of 49$)$ patients developed post-fine needle aspiration (FNA) pancreatitis.

Conclusion: Characteristic EUS-nCLE patterns can be consistently identified and improve the diagnostic accuracy of PCLs. These results support further investigations to optimize EUS-nCLE while minimizing adverse events.

\section{Study registration: NCT02516488}

Confocal Laser Endomicroscopy (CLE) is a promising new technology [6]. The technique involves real-time laser-assisted microscopic imaging of tissue where the system provides tissue sequences with a high resolution facilitating in vivo histopathology [7]. EUS-guided needle-based CLE ( $\mathrm{nCLE}$ ) is emerging as a capable technique with significant potential [8-10]. Imaging data from three clinical trials is currently the reference standard for nCLE imaging [7-9,11]. The diagnosis of either intraductal papillary mucinous neoplasms (IPMNs) or mucinous cystic neoplasms ( $\mathrm{MCNs}$ ) is indicated by the presence of finger-like papillae (specificity $100 \%$ ) and single band-like epithelium, respectively [8-10]. The specificity for diagnosing serous cystadenomas (SCAs) neared $100 \%$ when a characteristic "superficial vascular network" pattern was visualized [810]. The criterion for diagnosis of pseudocysts 
(bright particles, representing inflammatory cells against a black background [lack of epithelial lining or vascularity]) was confirmed in a recent study [8]. Endomicroscopy features of other rare types of PCLs including lymphoepithelial cysts, cystic-neuroendocrine tumors, retention cysts, and cystic degeneration of metastatic lesions need further characterization and validation [8].

More recently, a prospective external validation study (33 patients; 9 with surgical histopathology) from Europe demonstrated a "substantial" global intraobserver agreement (IOA) and diagnostic accuracy of $94 \%$ for mucinous PCLs [8]. In contrast, in another external validation study from the United States (15 patients; 3 with surgical histopathology), the IOA was "poor" to "fair" and the mean diagnostic accuracy was $46 \%$ for type of PCL [12]. A notable limitation for these and other preceding studies evaluating nCLE has been the low volume (6\% to $27 \%$ ) of patients who underwent surgical resection of PCLs for the "gold-standard" in diagnostic histopathology [7-11]. Another important drawback has been the absence of intraobserver reliability $[8,9$, 12].

Thus, we reviewed our institution's experience with EUS-guided nCLE for evaluation of PCLs to validate previously described criteria and analyze their diagnostic accuracy in differentiating mucinous from non-mucinous PCLs in a consecutive series of patients where a majority underwent surgical resection.

\section{Methods}

\section{Study design and population}

Our Institutional Review Board approved the following study. We performed a retrospective analysis of the EUS database and included all consecutive subjects who underwent EUS-guided nCLE for evaluation of PCLs from June 2013 to December 2015. A portion of the database included subjects $(n=5)$ who were prospectively enrolled in the INDEX study (Comparison of confocal laser endomicroscopic IN vivo Diagnosis and EX vivo examination against surgical histopathology of cystic pancreatic lesions; ClinicalTrials.gov NCT02516488). Our criteria for using EUSnCLE included: (a) subject age of 18 years or older, and (b) a PCL lesion size of $\geq 20 \mathrm{~mm}$ being evaluated for surgical removal based on recommended international consensus guidelines [3]. Exclusion criteria were: (a) pregnancy, (b) coagulopathy, and (c) known allergy to fluorescein.

\section{EUS-nCLE image acquisition}

All EUS examinations were performed using a linear echoendoscope (Olympus America, Center Valley, Pennsylvania, United States). While most EUS examinations were performed with intravenous propofol based sedation under the direction of an anesthesiologist, elective intubation for protection of airway in a few high risk patients was performed at the discretion of the supervising anesthesiologist. Fluorescein $(5 \mathrm{~mL}$ of $10 \%$ fluorescein sodium) was intravenously injected immediately before CLE imaging. The cystic lesion was penetrated under EUS guidance using 1 passage of a 19-gauge Flex (nitinol) needle (Boston Scientific, Natick, Massachusetts, United States). The preloaded AQ-Flex nCLE miniprobe (Cellvizio, Mauna Kea Technologies, Paris, France) was then advanced through the locking device into the 19-gauge (g) needle. The tip of the probe was advanced until it opposed the intracystic epithelium. Intracystic endomicroscopic images (video) were captured for 5 to 10 minutes with permissi-

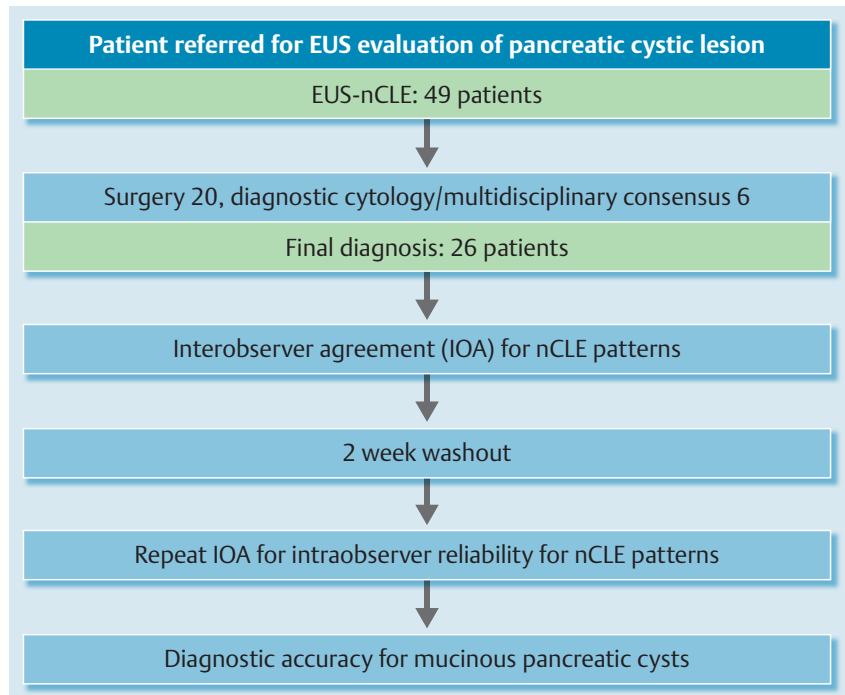

Fig. 1 Study flow chart. EUS-nCLE: endoscopic ultrasound guided needle based confocal laser endomicroscopy. IOA: interobserver agreement.

ble angulation of the 19-g needle. Next, the AQ-Flex probe was withdrawn from the 19-g needle and the locking device was removed. A syringe with negative suction was then attached to the proximal end of the 19-g needle. The cyst fluid was aspirated after acquisition of nCLE images. A quinolone (IV) was administered on the day of the procedure, then for 3 additional days (oral).

\section{Data collection}

Clinical variables of relevance including patient demographics, laboratory data, and clinical history were collected using a standardized data collection form. A radiologist (ZS) independently reviewed all cross-sectional imaging. Imaging data were compiled with those from EUS to describe: location, number, and size of the PCLs, lesion characteristics, evidence of dilation of the main pancreatic duct (MPD), and presence of communication with the MPD.

One gastroenterologist (SK) assisted by one gastrointestinal pathologist (BS), not blinded to the final diagnosis, reviewed the nCLE video records to select high yield image sequences. Since the nCLE video captures included a significant burden of low yield intracystic non-epithelial images, they were edited to shorter durations to best represent the PCL epithelium.

\section{Interobserver agreement and intraobserver reliability}

Six blinded, nCLE-naïve independent observers participated in the study. Three (SE, SM, JW) had completed fellowships in advanced endoscopy, two (DC, $\mathrm{PH})$ were medical pancreatologists (i.e., did not practice EUS) and one (AM) was a first year gastroenterology fellow. Teaching slides (30-slide Microsoft PowerPoint 2013; Microsoft Inc., Redmond, Washington, United States) with hand drawn schematics, nCLE images, and nCLE videos, were provided to the observers immediately preceding both phases of the interobserver agreement (IOA) study. The nCLE image videos used for the teaching sessions were different from the cases included in the study (early learning cases at our institute). The IOA study was performed in two steps with a 2-week washout ( $\bullet$ Fig. 1 ) using the same set of nCLE videos but with a different order of images. 

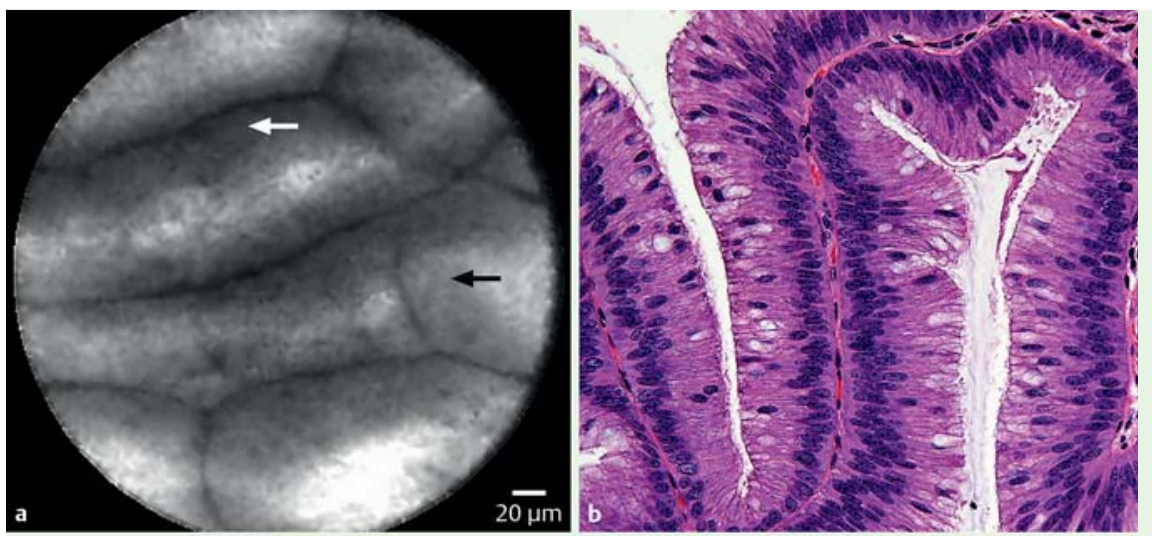

Fig. 2 EUS-guided needle based confocal endomicroscopy of intraductal papillary mucinous neoplasms (IPMN): “Finger-like” papillary projections representing the central fibrovascular core (black arrows) and overlying epithelium (white arrows) of the papillae. a, $\mathbf{c}$ In vivo EUS-nCLE imaging of branch duct IPMN. b, d surgical histopathology: $\mathbf{b}$, magnification $\times 40$; $d$, magnification $\times 10$.
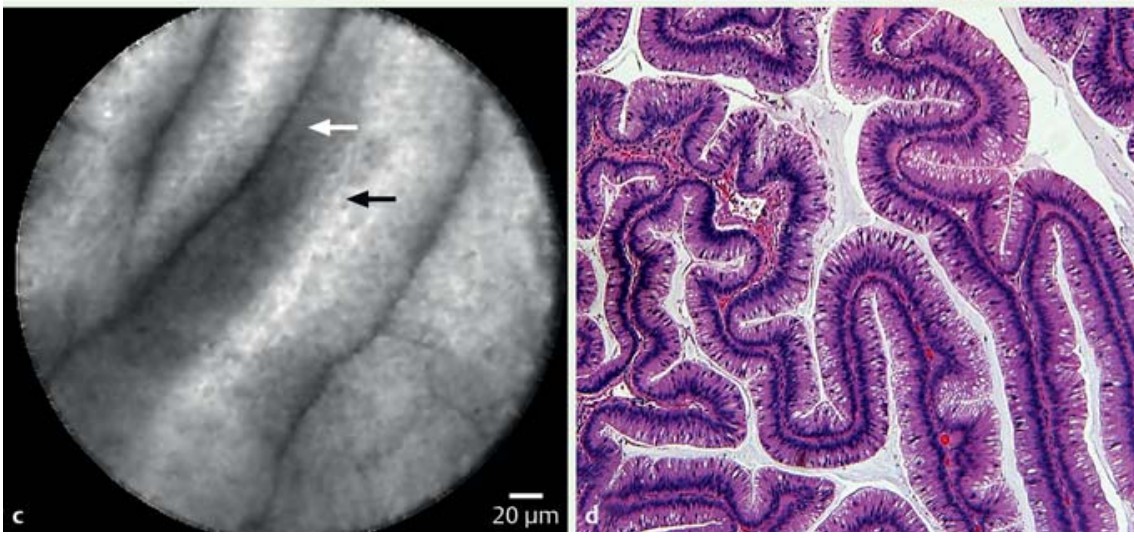

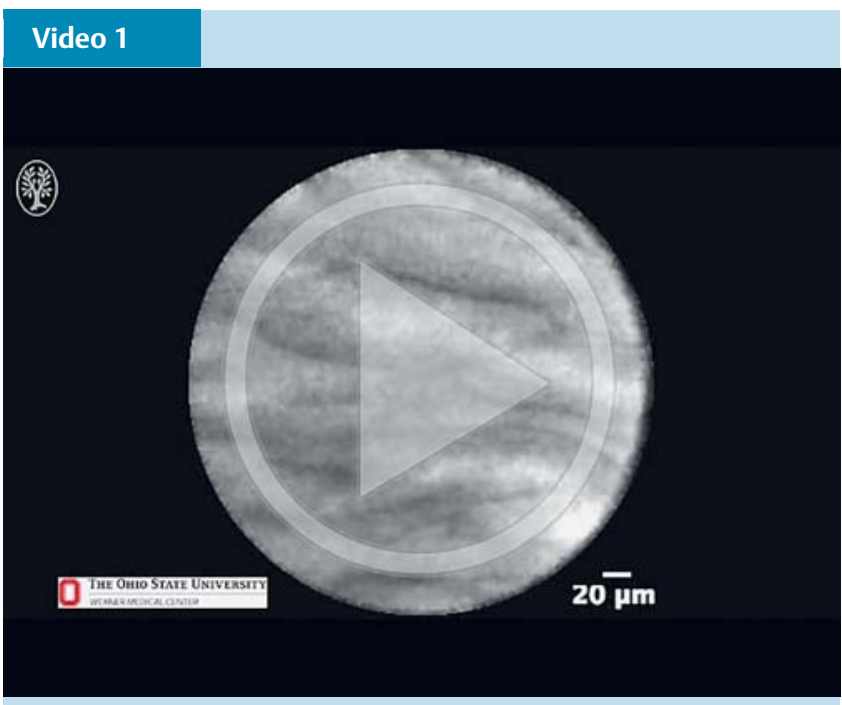

EUS-guided needle based confocal endomicroscopy of intraductal papillary mucinous neoplasms: "Finger-like" papillary projections representing the central fibrovascular core and overlying epithelium of the papillae. Online content including video sequences viewable at: http://dx.doi.org/10.1055/s0042-116491

The six observers independently reviewed the 26 EUS-nCLE videos (one from each patient) and categorically documented the presence or absence of the described nCLE image patterns of PCLs [7-11]. These characteristics included:

(a) Papillae or epithelial bands: Papillae ( Fig. 2; Video 1) of IPMN are finger-like projections of variable length consisting of an overlying epithelium and underlying vascular core. Nuclei do not absorb fluorescein, which contributes to the darkness of the epithelium. The vascular core absorbs fluorescein and appears bright. Single or multiple epithelial bands ( Fig.3; $\square$ Video 2 ) of MCN are observed without a papillary configuration. These bands demonstrated layering or a horizon-type configuration. (b) Fern-pattern of vascularity: This was previously described as a "superficial vascular network" of SCAs $[9,13]$. In this pattern, there is a concentrated network of parallel vessels emanating from a central vessel similar to a fern-leaf ( $\bullet$ Fig. 4; $\bullet$ Video 3 ).

(c) Bright particles against a dark background: Inflammatory cells, especially macrophages have autofluorescence and are observed as "bright particles" against a dark background ( $\bullet$ Fig. 5 a; - Video 4) [14]. These are mostly seen in pseudocysts, and some MCNs with chronic inflammation. However, unlike MCNs, pseudocysts do not have any epithelial bands [8]. Furthermore, pseudocysts demonstrate a completely dark background due to lack of a true wall and associated vascularity.

(d) After reviewing each nCLE video, the observers also categorically documented if the cyst was a mucinous versus non-mucinous PCL. The presence of papillae or epithelial bands and the absence of a fern-pattern of vascularity or features of a pseudocyst was consistent with a mucinous PCL.

\section{Final diagnosis}

All patients had been previously discussed in multidisciplinary pancreatic tumor board conferences. A gastrointestinal pathologist (BS) reviewed surgical pathology.

A total of 20 patients underwent surgical resection ( Table 1 ). Supplement 1 discusses the diagnostic details of patients without surgical histopathology ( $n=6 ; 3$ SCAs and 3 pseudocysts). For the three patients with pseudocysts, a definitive diagnosis was reached by cytology and consensus review at a multidisciplinary tumor board meeting. These patients presented with a history of alcoholism or hypertriglyceridemia or recent acute pancreatitis, and imaging studies, cyst fluid analysis, and cytology were diag- 

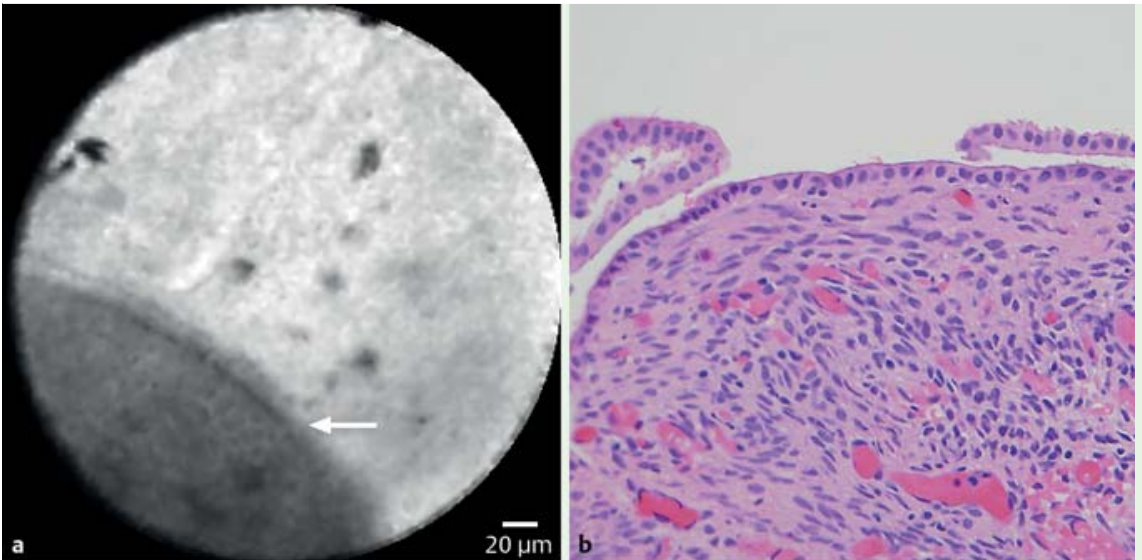

Fig. 3 EUS-guided needle based confocal endomicroscopy of mucinous cystic neoplasm (MCN). a, b MCN with low grade dysplasia. EUS-nCLE image (a) demonstrates a solitary epithelial band (white arrow) correlating with representative histology (b, magnification $\times 10$ ). c, d MCN with high grade dysplasia. EUS-nCLE image (c) reveals a thicker epithelial band (white arrow) relating to representative histology (d, magnification $\times 40)$.
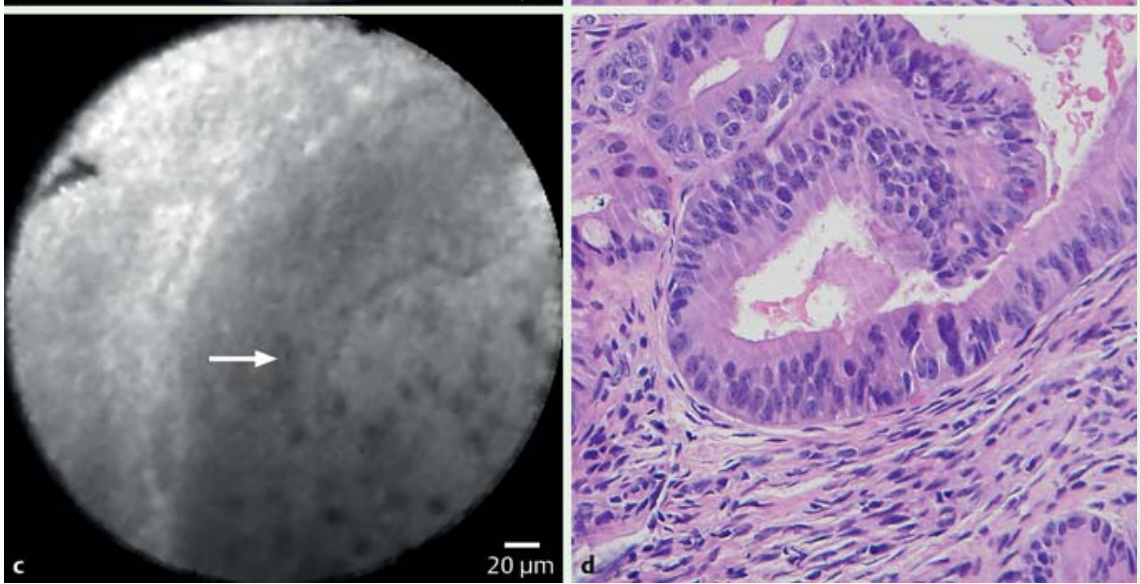

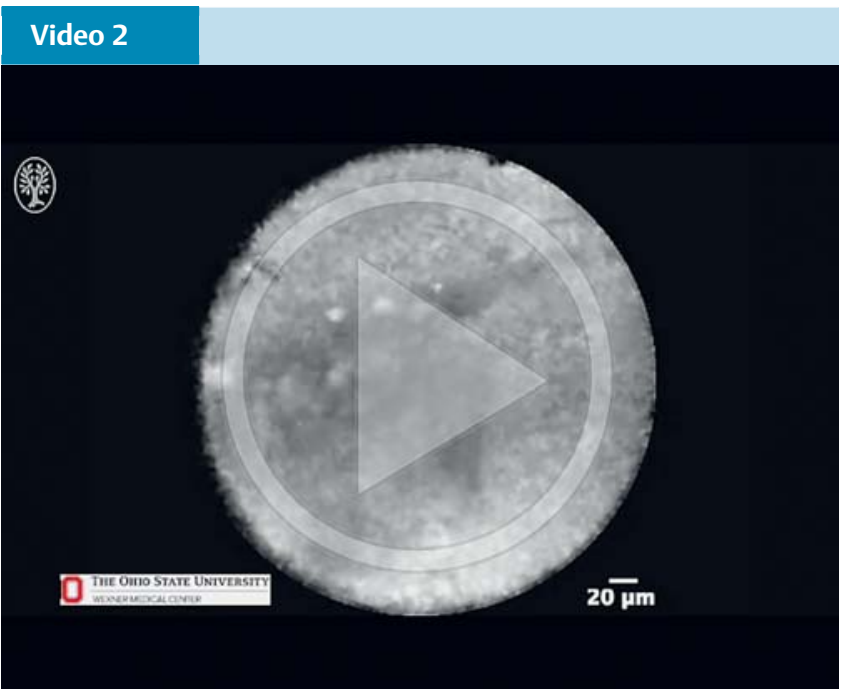

EUS-guided needle based confocal endomicroscopy of mucinous cystic neoplasm demonstrates multiple epithelial bands. These bands demonstrate layering or a horizon-type configuration. Online content including video sequences viewable at: http://dx.doi.org/10.1055/s-0042-116491

nostic of pseudocysts. Furthermore, all three subjects demonstrated a decrease in size of the cyst during a follow-up of $\geq 1$ year.

Three subjects were diagnosed with SCA on the basis of consensus review at a multidisciplinary tumor board meeting ( 3 pancreatobiliary surgeons, 4 advanced endoscopists, 1 radiologist), which involved review of demographics, clinical features, cross- sectional imaging studies, and EUS characteristics, and an "uneventful follow-up > 1 year". While the aspirate from endoscopic ultrasound guided fine needle aspiration (EUS-FNA) was of no yield (no fluid or cellularity) in two patients, one patient had a CEA of $0.5 \mathrm{ng} / \mathrm{dL}$.

\section{Statistical analysis}

Statistical analyses were performed using SPSS statistical package, version 21.0 (IBM Corporation, Somers, New York, United States). Continuous variables were compared with a $t$ test, while categorical variables were compared with either Chi-square test or Fisher's exact test, as appropriate. Statistical significance was defined as $P \leq 0.05$. Diagnostic accuracy parameters including area under receiver operating characteristic curve (AUC) of nCLE image patterns were calculated. The IOA for detection of nCLE image patterns was calculated using Fleiss' kappa (к) statistic (with 95\% confidence intervals $[\mathrm{CI}]$ ). The intraobserver reliability was calculated using Cohen's kappa (к) statistic. The interpretation of $\mathrm{K}$ values by Landis and Koch was used ( $<0$ : indicating no agreement; 0-0.20: slight; 0.21-0.40: fair; 0.41-0.60: moderate; $0.61-0.80$ : substantial; and $0.81-1$ : almost perfect agreement) [15].

Sensitivity, specificity, positive predictive value (PPV), and negative predictive value (NPV) for the detection of mucinous PCLs were calculated for each observer in both phases of the IOA study. In addition, cumulative values were calculated by averaging the results from all six observers at each phase. The cumulative values were compared for statistically significant difference using paired $t$ tests.

The only variable with missing data was cyst fluid CEA ( $\bullet$ Table 1 ). Multiple imputation was not performed and these data were an- 

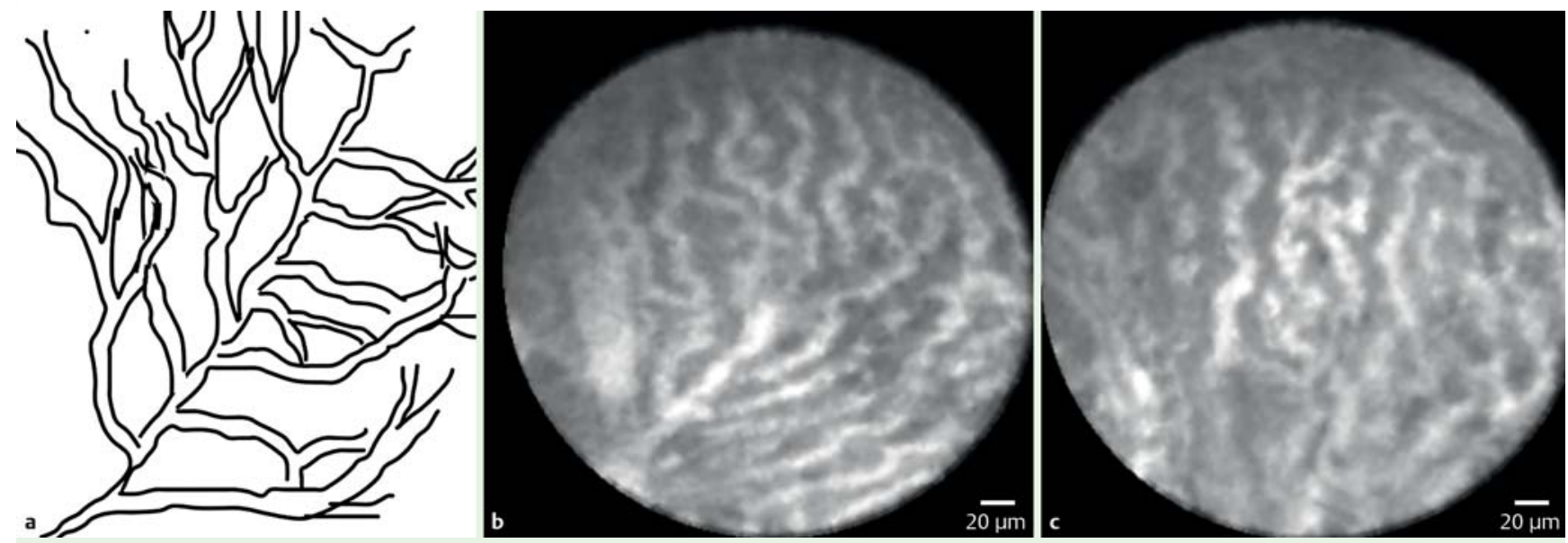

Fig. 4 EUS-guided needle based confocal endomicroscopy (nCLE) in pancreatic cystic lesions. a Schematic diagram of the "fern-pattern" of vascularity in serous cystadenoma. b, c nCLE images of the "fern-pattern" of vascularity.

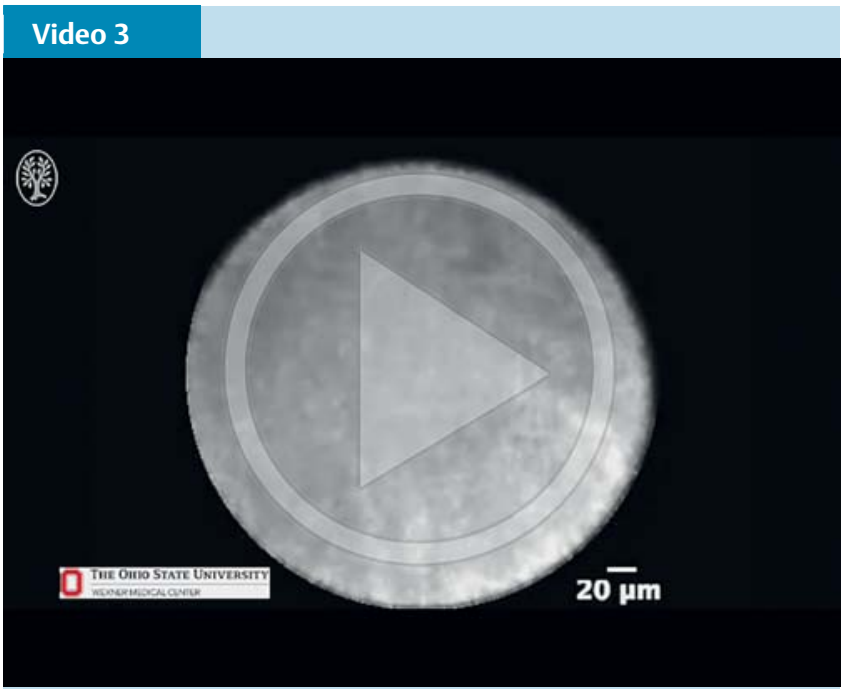

EUS-guided needle based confocal endomicroscopy of serous cystadenoma demonstrates a concentrated network of parallel vessels emanating from a central vessel similar to a fern leaf. We termed this as "fern-pattern" vascularity. This has been called "superficial vascular network" in earlier publications. Online content including video sequences viewable at: http://dx.doi. org/10.1055/s-0042-116491

alyzed "as is" to represent actuality. A sample size of 26 patients with three observations per patient and six independent observers would achieve an $80 \%$ power to detect an IOA of 0.61 ("substantial" or above).

\section{Results}

$\nabla$

\section{Study cohort}

A total of 49 patients underwent EUS-nCLE during the study period. A total of 26 patients ( 16 female; mean age $54.8 \pm 12.6$ years [ \pm standard deviation]) had a definitive diagnosis and were selected for further study ( $\bullet$ Fig. 1 ). Mean size of PCLs was $31.7 \pm 12.8 \mathrm{~mm}$. Mean duration of nCLE imaging during EUS was $6.1 \pm 2.8$ minutes. Most cysts were located in the tail $(13,50 \%)$, compared to the neck/body $(8,30.8 \%)$, or head/uncinate region $(5,19.2 \%)$. The only adverse event observed during the study was acute pancreatitis in $3 / 49(6.1 \%)$ subjects.
Demographics, clinical features, EUS findings, final diagnoses, and follow-up duration for the 26 patients are displayed in $1 \mathrm{Ta}-$ ble 1. A pathological diagnosis was available in 23 (88.4\%) patients (surgery, $n=20$; diagnostic cytology, $n=3$ ); three patients with SCAs did not undergo surgical resection. The mean followup period for the six subjects (3 SCA, 3 pseudocysts) with nonsurgical management was $433.5 \pm 58.1$ days.

Cyst fluid CEA was available in $73.1 \%$ (19 of 26) of patients. A total of seven subjects did not have a cyst fluid CEA available, including: patients $(n=3)$ with IPMN having a markedly viscous aspirate, subjects $(n=2)$ with a dry aspirate in the setting of an SCA, subjects with a pasty aspirate (due to pseudocyst [ $n=1]$, and lymphoepithelial cyst $[n=1])$. The sensitivity, specificity, accuracy, and AUC of cyst fluid CEA (value $\geq 192 \mathrm{ng} / \mathrm{mL}$ ) for diagnosis of mucinous PCLs when available were 50\%, 43\%, 47\%, and 0.40 , respectively. The sensitivity, specificity, accuracy, and AUC of cytology for diagnosis of mucinous PCLs were 53\%, 64\%, 58\%, and 0.59 , respectively. An intracystic solid component was observed in $6(23.1 \%)$ subjects where 5 of them were IPMNs, and $1 \mathrm{MCN}$. Collectively, imaging of the cyst by MRI or EUS demonstrated thin intracystic septations in $50 \%$ of the cysts and a communication with the MPD was observed in all IPMN patients $(n=10)$. High grade dysplasia was observed in 6 of 10 IPMNs and 1 of 5 MCNs that were resected. A majority (50\%) of the IPMNs resected were of the gastric subtype. Six of the 10 IPMNs resected were of the mixed-duct type while the rest were all branch-duct IPMNs.

\section{Interobserver agreement}

The IOA was analyzed for detection of nCLE patterns in two phases separated by a 2-week wash-out ( $\bullet$ Table 2 ). In the first phase of the study, there was "substantial" IOA for detection of all nCLEimage patterns including papillae or epithelial bands, bright particles on a dark background, and fern-pattern of vascularity. The IOA for differentiating mucinous vs. non-mucinous PCL was "substantial" ( $\mathrm{K}=0.67,95 \% \mathrm{CI} 0.57,0.77$ ). In the second phase of the study, the IOAs for each of the three nCLE-image patterns improved slightly, however, without a change in the interpretation which remained at "substantial" agreement ( $\bullet$ Table 2). The IOA for diagnosis of a mucinous PCL $(\mathrm{K}=0.66,95 \% \mathrm{CI} 0.56,0.76)$ remained unchanged in the second phase of the study. 

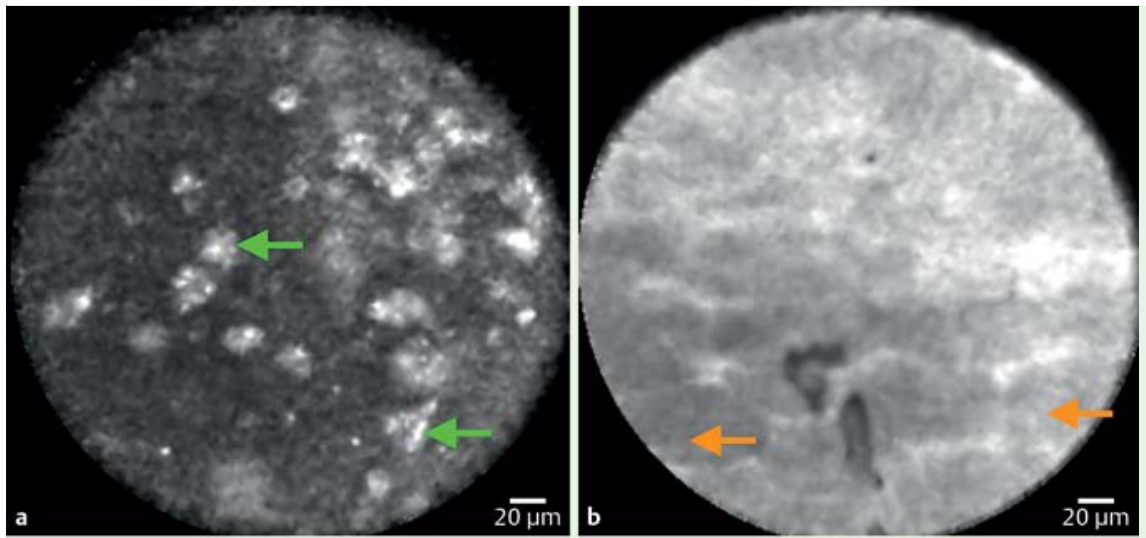

Fig. 5 EUS-guided needle based confocal endomicroscopy of pancreatic cysts. a Pseudocysts lack an underlying epithelium and demonstrate multiple clusters of bright, floating, heterogeneous particles representing inflammatory debris and cells (autofluorescent macrophages: green arrows). Due to lack of vascularity, the background is dark. $\mathbf{b}$ Lymphoepithelial cyst revealing squamous type epithelium (orange arrows). c Epidermoid cyst also revealing squamous type epithelial cells (yellow arrows). d Lymphoepithelial cyst with abundant keratin (blue arrows).

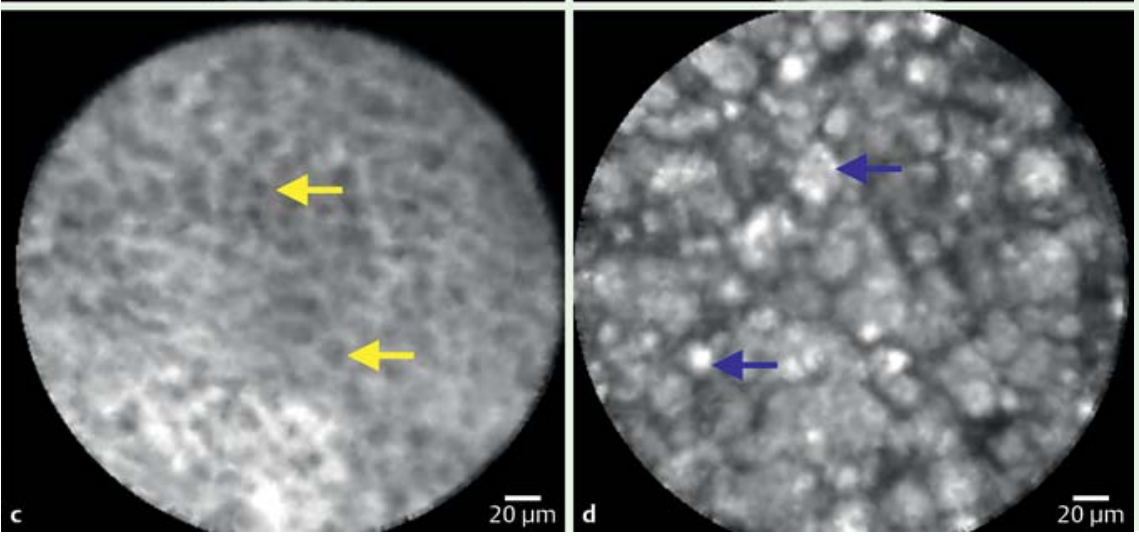

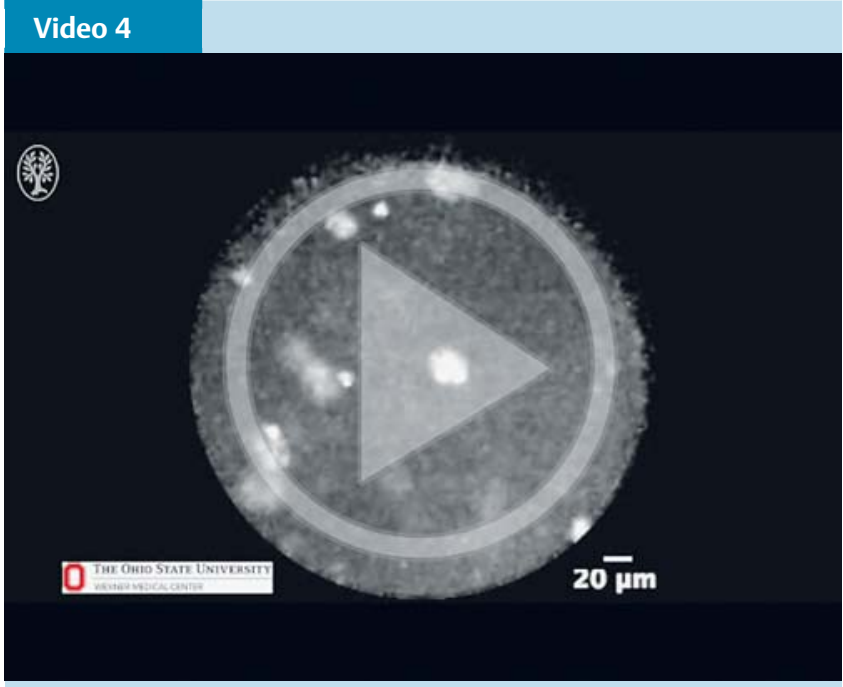

EUS-guided needle based confocal endomicroscopy of pseudocysts: Bright particles representing autofluorescent inflammatory macrophages set against a dark background. Online content including video sequences viewable at: http://dx.doi.org/10.1055/s-0042-116491

\section{Intraobserver reliability}

The mean intraobserver reliability was "almost perfect" $(\mathrm{K}=0.81$ \pm 0.18 ) among EUS physicians and "substantial" among non-EUS physicians; overall the group reached "substantial" $(\mathrm{K}=0.78 \pm$ $0.13)$ reliability for diagnosing mucinous PCLs ( 0 Table 3 ). The mean intraobserver reliability was "substantial" for detection of "bright particles with dark background" $(\mathrm{K}=0.78 \pm 0.12)$ and "fern-pattern" vascularity $(\mathrm{k}=0.68 \pm 0.20)$. The spectrum of intraobserver reliability ranged from "substantial" to "almost perfect" for detection of "papillae" and "bright particles with dark background" while being "moderate" to "almost-perfect" for "fern-pattern" vascularity.

\section{Diagnosis of mucinous pancreatic cystic lesions}

The overall cumulative sensitivity, specificity, positive predictive value, negative predictive value, accuracy, and AUC for diagnosis of a mucinous PCL were $94 \%, 82 \%, 88 \%, 92 \%, 89 \%$, and 0.88 , respectively. These cumulative measures of diagnostic accuracy in differentiating mucinous PCLs remained mostly unchanged in the second phase of the IOA study ( $\bullet$ Table 4 ). There was no statistically significant difference comparing cumulative measures of diagnostic accuracy between physicians performing EUS vs. not (Supplement 2).

\section{EUS-nCLE findings in squamous epithelial-lined cysts}

Collectively termed squamous epithelium-lined cysts of the pancreas, these benign cysts included lymphoepithelial cysts and epidermoid cysts ( $\bullet$ Table 1 ). A flat, scale-like epithelium ( $\odot$ Fig. 5 b, c) was observed in both. One of the two lymphoepithelial cysts demonstrated keratinaceous debris $(\boldsymbol{\bullet}$ Fig. $\mathbf{5 d}$ ). Neither of these cysts revealed papillae or epithelial bands.

\section{Discussion}

$\nabla$

The primary aim of our study was to validate recent diagnostic characteristics of EUS-nCLE in differentiating mucinous PCLs. We demonstrate that EUS-nCLE provides in vivo diagnostic imaging of large $(\geq 20 \mathrm{~mm})$ pancreatic cysts with potentially higher accuracy than the current standard of practice. Gastroenterologists with pre-trial nCLE image training who were otherwise naive to CLE and blinded to the patient information can reliably identify these diagnostic image patterns with substantial inter- 


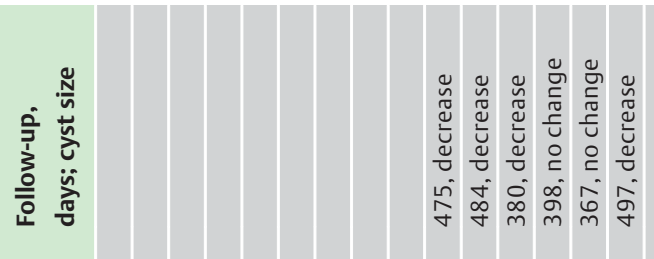

के ने ते ने के ते के ने ते

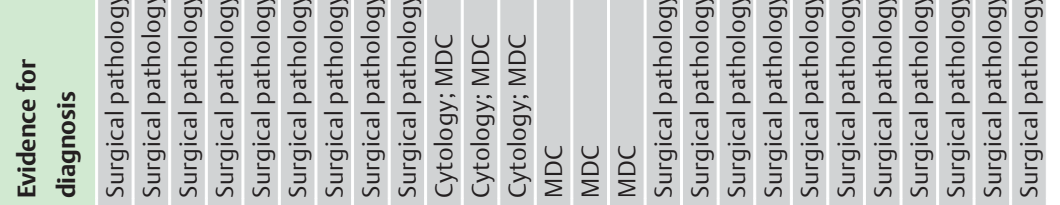

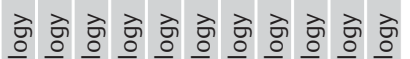

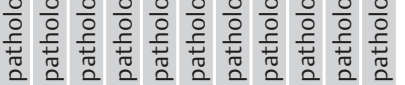

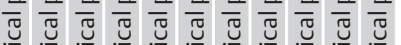

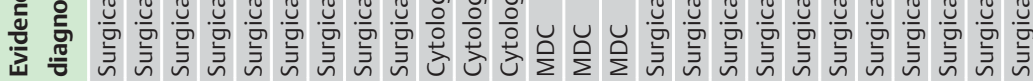

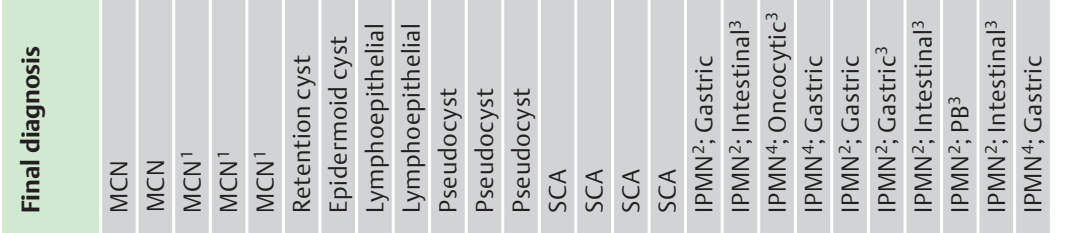

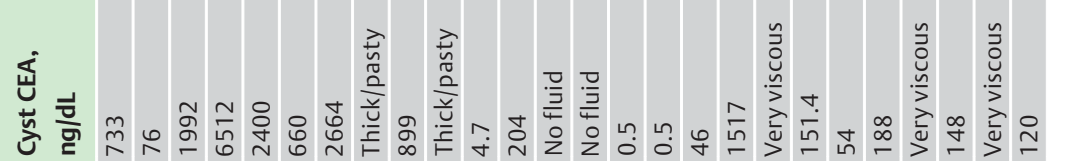
$\stackrel{\dot{3}}{3}$

응

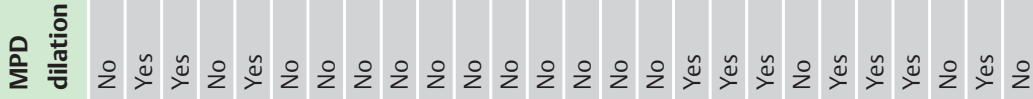
$\frac{n}{n}$

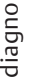

:

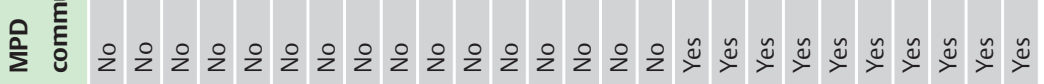

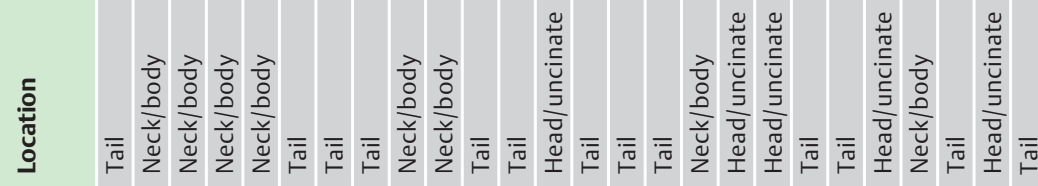
言

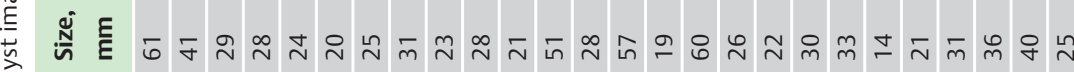
这

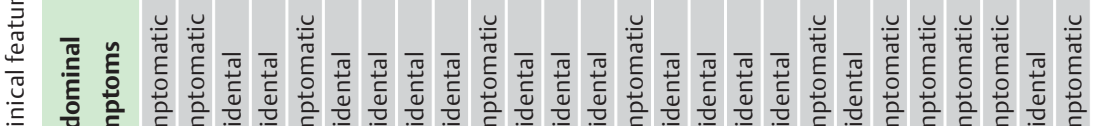

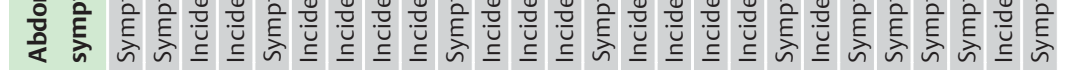
芦

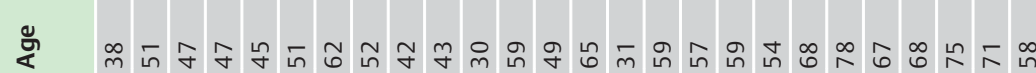


Table 2 Interobserver agreement of six observers for the detection of nCLEimage patterns and diagnosis of pancreatic cystic lesions.

\begin{tabular}{|llllll|} 
& \multicolumn{2}{c}{$\mathbf{1}^{\text {st }}$ phase IOA study } & \multicolumn{2}{c|}{$\mathbf{2}^{\text {nd }}$ phase IOA study } \\
\hline nCLE image pattern & Fleiss K & $95 \% \mathrm{Cl}$ & Fleiss K & $95 \% \mathrm{Cl}$ \\
\hline $\begin{array}{l}\text { Papillae or epithelial } \\
\text { bands }\end{array}$ & 0.63 & $0.53,0.73$ & 0.65 & $0.55,0.75$ \\
\hline $\begin{array}{l}\text { Dark background } \\
\text { with bright particles }\end{array}$ & 0.71 & $0.61,0.81$ & 0.79 & $0.69,0.89$ \\
\hline $\begin{array}{l}\text { Fern-leaf } \\
\text { Mucinous cyst (IPMN }\end{array}$ & 0.62 & $0.52,0.72$ & 0.70 & $0.60,0.80$ \\
\hline \begin{tabular}{l} 
or MCN) \\
\hline
\end{tabular} & 0.67 & $0.57,0.77$ & 0.66 & $0.56,0.76$ \\
\hline
\end{tabular}

$\mathrm{Cl}$ : confidence interval; IOA: interobserver agreement; IPMN: intraductal papillary mucinous neoplasm; MCN: mucinous cystic neoplasm; 2nd IOA study was conducted 2 weeks (wash-out period) after the 1st study.

Landis and Koch interpretation IOA and intrarater reliability: <0, no agreement; $0-0.20$, slight; $0.21-0.40$, fair; $0.41-0.60$, moderate; $0.61-0.80$, substantial; and $0.81-1$, almost perfect agreement.

and intraobserver agreement. We included consecutive patients encompassing rare types of PCLs (squamous epithelial cysts) to best represent the referral community. To our knowledge, this is the largest study describing nCLE findings in definitively diagnosed PCLs. These promising findings require additional investi- gation including validation in larger multicenter studies for the application of EUS-nCLE in the management of PCLs.

A summary of current literature evaluating nCLE-guided diagnosis of PCLs is given in $\bullet$ Table 5. The proportion of patients with surgical histopathology in the INSPECT, DETECT, and CONTACT trials is $21.2 \%$ ( 14 of 66 ), $6.7 \%$ ( 2 of 30 ), and $27.3 \%$ (9 of 33 ), respectively. In another recent study evaluating IOA for EUSnCLE patterns, only $20 \%$ ( 3 of 15 ) of patients had undergone surgical resection of PCLs. Compared to these trials, our study consisted of $77 \%$ (20 of 26 ) of patients with confirmed histopathology. Moreover, all of the mucinous PCLs had diagnostic goldstandard and the only cysts without surgical histopathology were SCAs and pseudocysts where in fact surgery is not recommended.

Based on published literature (including the current study), several conclusions can be made with regard to diagnostic nCLE-patterns of PCLs. The presence of either papillary epithelium or epithelial bands has high diagnostic accuracy for mucinous PCLs. Although pre-surgical differentiation between IPMN and MCN does not significantly impact patient management, the epithelial bands in MCNs lack a papillary conformation and sometimes demonstrate a fan-like or horizon-type layering, whereas papillae in IPMNs are complete and identified with lesser effort. Some

Table 3 Intraobserver reliability of six observers for detection of nCLE-image patterns and diagnosis of pancreatic cystic lesions.

\begin{tabular}{|c|c|c|c|c|c|c|c|}
\hline & \multicolumn{7}{|c|}{ Independent blinded nCLE-naïve observers (Cohen's к) } \\
\hline & 1 & 2 & 3 & 4 & 5 & 6 & Mean \pm SD \\
\hline nCLE image pattern & \multicolumn{3}{|c|}{ EUS physicians } & \multicolumn{4}{|c|}{ Non-EUS physicians } \\
\hline Papillae or epithelial bands & 0.91 & 0.85 & 0.64 & 0.69 & 0.68 & 0.77 & $0.76 \pm 0.11$ \\
\hline Dark background with bright particles & 0.61 & 0.82 & 0.86 & 0.65 & 0.91 & 0.83 & $0.78 \pm 0.12$ \\
\hline Fern-pattern vascularity & 1.00 & 0.71 & 0.46 & 0.71 & 0.46 & 0.71 & $0.68 \pm 0.20$ \\
\hline Mucinous cyst (IPMN or MCN) & 0.91 & 0.92 & 0.61 & 0.69 & 0.84 & 0.69 & $0.78 \pm 0.13$ \\
\hline
\end{tabular}

nCLE: needle-based confocal laser endomicroscopy; SD: standard deviation; IPMN: intraductal papillary mucinous neoplasm; MCN: mucinous cystic neoplasm. 2nd phase of intraobserver (IOA) study was conducted 2 weeks (wash-out period) after the 1st study.

Landis and Koch interpretation IOA and intrarater reliability: $<0$, no agreement; $0-0.20$, slight; $0.21-0.40$, fair; $0.41-0.60$, moderate; $0.61-0.80$, substantial; and 0.81 - 1 , almost perfect agreement.

Table 4 Measures of diagnostic accuracy in differentiating a mucinous pancreatic cystic lesion using EUS-guided needle based confocal laser endomicroscopy: comparison between the two phases of interobserver agreement study.

\begin{tabular}{|c|c|c|c|c|c|c|c|}
\hline & Observers & Sensitivity (\%) & Specificity (\%) & PPV (\%) & NPV (\%) & Accuracy (\%) & ROC \\
\hline \multicolumn{8}{|l|}{ IOA Phase 1} \\
\hline \multirow[t]{3}{*}{ EUS physicians } & 1 & 100 & 100 & 100 & 100 & 100 & 1.0 \\
\hline & 2 & 100 & 73 & 83 & 100 & 89 & 0.86 \\
\hline & 3 & 93 & 64 & 78 & 88 & 81 & 0.79 \\
\hline \multirow[t]{4}{*}{ Non-EUS physicians } & 4 & 87 & 91 & 93 & 83 & 89 & 0.89 \\
\hline & 5 & 93 & 73 & 82 & 89 & 85 & 0.83 \\
\hline & 6 & 93 & 91 & 93 & 91 & 92 & 0.92 \\
\hline & Mean \pm SD & $94 \pm 5$ & $82 \pm 14$ & $88 \pm 8$ & $91 \pm 7$ & $88 \pm 6$ & $0.88 \pm 0.06$ \\
\hline \multicolumn{8}{|l|}{ IOA Phase 2} \\
\hline \multirow[t]{3}{*}{ EUS physicians } & 1 & 93 & 100 & 100 & 92 & 96 & 0.97 \\
\hline & 2 & 100 & 82 & 88 & 100 & 92 & 0.91 \\
\hline & 3 & 100 & 54 & 75 & 100 & 81 & 0.77 \\
\hline \multirow[t]{4}{*}{ Non-EUS physicians } & 4 & 93 & 82 & 88 & 90 & 89 & 0.88 \\
\hline & 5 & 87 & 82 & 87 & 82 & 85 & 0.84 \\
\hline & 6 & 93 & 91 & 93 & 91 & 92 & 0.92 \\
\hline & Mean \pm SD & $94 \pm 5$ & $82 \pm 15$ & $89 \pm 8$ & $93 \pm 7$ & $89 \pm 5$ & $0.88 \pm 0.07$ \\
\hline $\begin{array}{l}P \text { value: IOA } \\
\text { Phase } 1 \text { vs. } 2\end{array}$ & & 1.0 & 0.96 & 0.85 & 0.84 & 0.86 & 1.0 \\
\hline
\end{tabular}

SD: standard deviation; PPV: positive predictive value; NPV: negative predictive value; ROC: receiver operating characteristic curve; 2nd phase of intraobserver (IOA) study was conducted 2 weeks (wash-out period) after the 1st study. 
Table 5 Outcome, diagnostic accuracies, and risk of pancreatitis for major trials investigating role of endoscopic ultrasound guided needle based confocal laser endomicroscopy in diagnosis of pancreatic cystic lesions.

\begin{tabular}{|llllllll|}
\hline Study title and reference & Study outcome & Patients, $\mathbf{n}$ & Surgery, $\mathbf{n}(\%)$ & Sensitivity, \% & Specificity, \% & Accuracy, \% & Pancreatitis rate, \% \\
\hline INSPECT [13] & Neoplastic cyst & 66 & $14(21.2 \%)$ & 59 & 100 & 71 & 3 \\
\hline DETECT [7] & Mucinous cyst & 30 & $2(6 \%)$ & 80 & 100 & 89 & 6.6 \\
\hline CONTACT-1 [11] & SCA & 31 & $7(22.5 \%)$ & 69 & 100 & 87 & 3.2 \\
\hline CONTACT-2 [10] & Mucinous cyst & 33 & $9(27.3 \%)$ & 91 & 95 & 94 & Same as CONTACT-1 \\
\hline Current study (INDEX) & Mucinous cyst & 26 & $20(76.9 \%)$ & 94 & 82 & 89 & 6.1
\end{tabular}

DETECT study: High certainty $(n=18)$ patients included in analysis of diagnostic accuracy. SCA: Serous cystadenoma.

MCNs (40\% in this series) demonstrated evidence of chronic inflammation with visualization of "bright" fluorescent inflammatory cells [16].

For the diagnosis of SCAs, detection of the fern-pattern of vascularity (superficial vascular network) is highly specific $[8,9,13]$. In the absence of this pattern, the sensitivity for diagnosis of the SCAs remains low (69\% to 75\%) [8,9]. Since the epithelium of oligocystic SCAs can become denuded, they can be difficult to distinguish when the characteristic vascular pattern is not observed [16]. The nCLE criteria for diagnosis of pseudocysts have been validated in a recent study [8]. Pseudocysts do not have an epithelium, thus no vasculature is observed [16]. Distinct, multiple autofluorescent inflammatory cells are observed against a dark background during nCLE.

In CONTACT-2nd phase, IOAs were "almost perfect" for vascular pattern of SCAs, "fair" for epithelial pattern of MCNs, "moderate" for papillae in IPMNs, and "perfect" for pseudocysts. The global IOA was "substantial". Our study yielded a similarly favorable IOA with "substantial" agreement for identification of epithelial and vascular image patterns and for discrimination of mucinous cysts. In addition, we conducted IOAs in two phases demonstrating overall "substantial" intraobserver reliability. Our results are in sharp contrast with a recent study where videos from 15 nCLE patients were reviewed for features that were not particularly diagnostic of a PCL type. This study, however, had multiple methodological issues including the relatively short duration of nCLE video capture (mean $<2$ minutes), only three patients with surgical histopathology, absence of evaluation for intraobserver reliability, and poor quality of nCLE images [12].

The risk of post-procedural acute pancreatitis from the three major trials utilizing nCLE for PCLs gives us an overall rate of $4.3 \%$ ( $\bullet$ Table 5) $[7,8,11]$. The highest risk was with the DETECT study (6.6\%) and, understandably, since the procedure involved longer needle access time for Spyglass cystoscopy and nCLE imaging. The reported risk of acute pancreatitis for 22-g and/or 25-g needles in PCLs is $2.4 \%$ [17]. The outer diameter of a standard 19-g needle is $1.07 \mathrm{~mm}$ (area $0.9 \mathrm{~mm}^{2}$ ). Comparatively, a standard 22-g needle has an outer diameter of $0.72 \mathrm{~mm}$ (area $0.41 \mathrm{~mm}^{2}$ ), half the area of the 19-g needle. Although studies comparing a 19-g to 22-g and/or 25-g needle for FNA of solid pancreatic lesions have not demonstrated any significant increase in the risk of post-procedural pancreatitis, this does not simulate similar risks when aspirating cystic lesions $[18,19]$. Several theories including duration of nCLE and manipulation of the elevator or scope-torque to access other areas of the cyst have been proposed to increase the risk of pancreatitis [7]. The risk of pancreatitis in our study was $6.1 \%$, including one subject who developed severe pancreatitis with extra pancreatic necrosis. However, it should be noted that this subject had a coughing spell during a critical portion of the procedure resulting in shearing injury by the needle. In the final study cohort $(n=26)$, patients with longer duration of image acquisition ( $8 \pm 2.1$ vs. $6.4 \pm 2.7$ minutes; $P=0.02$ ) were at increased risk for pancreatitis. This suggests that reducing the image acquisition time to 6 minutes or less may decrease the rate of adverse events.

There are obvious limitations to our study. First and foremost, this is a retrospective study with a small sample size and this compels a larger, prospective multicenter representation and external validation for generalizability. Second, the final diagnosis of 6 PCLs was by multidisciplinary consensus. A diagnosis of a non-mucinous PCLs was reached by multidisciplinary consensus and thus surgery was not performed. These patients continue to be followed at our institute (currently > 1 year of follow-up; Supplement 1 ). Third, only a portion of the intracystic epithelium can be sampled due to limitations at the point of needle puncture. Besides, improved technology in the CLE probes to permit utilization in a 22-g needle would permit a larger nCLE viewing area of PCLs, potentially decrease the risk of post-FNA pancreatitis, and likely translate to wider adoption by gastroenterologists. Fourth, we selected consecutive patients with cysts $>20 \mathrm{~mm}$ in size and those being evaluated for surgical resection; these factors introduce a potential for significant selection bias where an enriched population is being evaluated.

In conclusion, EUS-guided nCLE is a minimally invasive procedure that can potentially improve the preoperative diagnostic performance of PCLs allowing diagnosis of mucinous cysts with improved accuracy. This technology is currently used in a small number of academic centers. There is a low, albeit present, risk of post-procedural acute pancreatitis, which we believe can be reduced by limiting the time of image acquisition. EUS-nCLE is potentially complementary and can be combined with novel cyst fluid molecular markers, and a collective strategy may need to be explored for accurate diagnosis and risk stratification of pancreatic cystic lesions.

Competing interests: There are no relevant conflicts of interest to report for any author.

Institutions

${ }^{1}$ Section of Advanced Endoscopy, The Ohio State University Wexner Medical Center, Columbus, Ohio, USA

2 Section of Pancreatic Disorders, Division of Gastroenterology, Hepatology and Nutrition, The Ohio State University Wexner Medical Center, Columbus, Ohio, USA

${ }^{3}$ Department of Pathology, The Ohio State University Wexner Medical Center, Columbus, Ohio, USA

${ }^{4}$ Department of Radiology, The Ohio State University Wexner Medical Center, Columbus, Ohio, USA 


\section{Acknowledgments}

A portion of the database $(n=5)$ included subjects who were prospectively enrolled in the INDEX study (Comparison of confocal laser endomicroscopic IN vivo Diagnosis and EX vivo examination against surgical histopathology of cystic pancreatic lesions; ClinicalTrials.gov NCT02516488). The INDEX study is funded by the American College of Gastroenterology pilot research grant.

\section{References}

1 de Jong K, Nio CY, Hermans JJ et al. High prevalence of pancreatic cysts detected by screening magnetic resonance imaging examinations. Clin Gastroenterol Hepatol 2010; 8: 806-811

2 Laffan TA, Horton KM, Klein AP et al. Prevalence of unsuspected pancreatic cysts on MDCT. AJR Am J Roentgenol 2008; 191: $802-807$

3 Tanaka M, Fernandez-del Castillo C, Adsay V et al. International consensus guidelines 2012 for the management of IPMN and MCN of the pancreas. Pancreatology 2012; 12: 183-197

4 Valsangkar NP, Morales-Oyarvide V, Thayer SP et al. 851 resected cystic tumors of the pancreas: a 33-year experience at the Massachusetts General Hospital. Surgery 2012; 152: 4-12

5 Gaujoux S, Brennan MF, Gonen $M$ et al. Cystic lesions of the pancreas: changes in the presentation and management of 1,424 patients at a single institution over a 15-year time period. J Am Coll Surg 2011; 212: 590-600; discussion 600-593

6 Krishna SG, Lee JH. Appraisal of needle-based confocal laser endomicroscopy in the diagnosis of pancreatic cysts. World J Gastroenterol 2016; $22: 1701-1710$

7 Nakai Y, Iwashita T, Park do H et al. Diagnosis of pancreatic cysts: EUSguided, through-the-needle confocal laser-induced endomicroscopy and cystoscopy trial: DETECT study. Gastrointest Endosc 2015; 81: $1204-1214$

8 Napoleon B, Lemaistre AI, Pujol B et al. In vivo characterization of pancreatic cystic lesions by needle-based confocal laser endomicroscopy ( $\mathrm{nCLE}$ ): proposition of a comprehensive nCLE classification confirmed by an external retrospective evaluation. Surg Endosc 2016; 30: 2603 2612

9 Napoleon B, Lemaistre AI, Pujol B et al. A novel approach to the diagnosis of pancreatic serous cystadenoma: needle-based confocal laser endomicroscopy. Endoscopy 2015; 47: 26-32

10 Krishna SG, Swanson B, Conwell DL et al. In vivo and ex vivo needlebased confocal endomicroscopy of intraductal papillary mucinous neoplasm of the pancreas. Gastrointest Endosc 2015; 82: 571 -572

11 Konda VJ, Meining A, Jamil $L H$ et al. A pilot study of in vivo identification of pancreatic cystic neoplasms with needle-based confocal laser endomicroscopy under endosonographic guidance. Endoscopy 2013; 45: $1006-1013$

12 Karia K, Waxman I, Konda VJ et al. Needle-based confocal endomicroscopy for pancreatic cysts: the current agreement in interpretation. Gastrointest Endosc 2016; 83: 924-927

13 Modi RM, Swanson B, Muscarella P2nd et al. Novel techniques for diagnosis of serous cystadenoma: fern pattern of vascularity confirmed by in vivo and ex vivo confocal laser endomicroscopy. Gastrointest Endosc 2016: DOI 10.1016/j.gie.2016.07.015

14 Thiberville L, Salaun M, Lachkar $S$ et al. Confocal fluorescence endomicroscopy of the human airways. Proc Am Thorac Soc 2009; 6: 444 - 449

15 Landis JR, Koch GG. A one-way components of variance model for categorical data. Biometrics 1977; 33: 671-679

16 Volkan Adsay N. Cystic lesions of the pancreas. Mod Pathol 2007; 20 : $71-93$

17 de Jong K, Poley JW, van Hooft JE et al. Endoscopic ultrasound-guided fine-needle aspiration of pancreatic cystic lesions provides inadequate material for cytology and laboratory analysis: initial results from a prospective study. Endoscopy 2011; 43: 585-590

18 Ramesh J, Bang JY, Hebert-Magee $S$ et al. Randomized trial comparing the flexible 19G and 25G needles for endoscopic ultrasound-guided fine needle aspiration of solid pancreatic mass lesions. Pancreas 2015; 44: $128-133$

19 Song TJ, Kim JH, Lee SS et al. The prospective randomized, controlled trial of endoscopic ultrasound-guided fine-needle aspiration using 22G and 19G aspiration needles for solid pancreatic or peripancreatic masses. Am J Gastroenterol 2010; 105: 1739-1745 


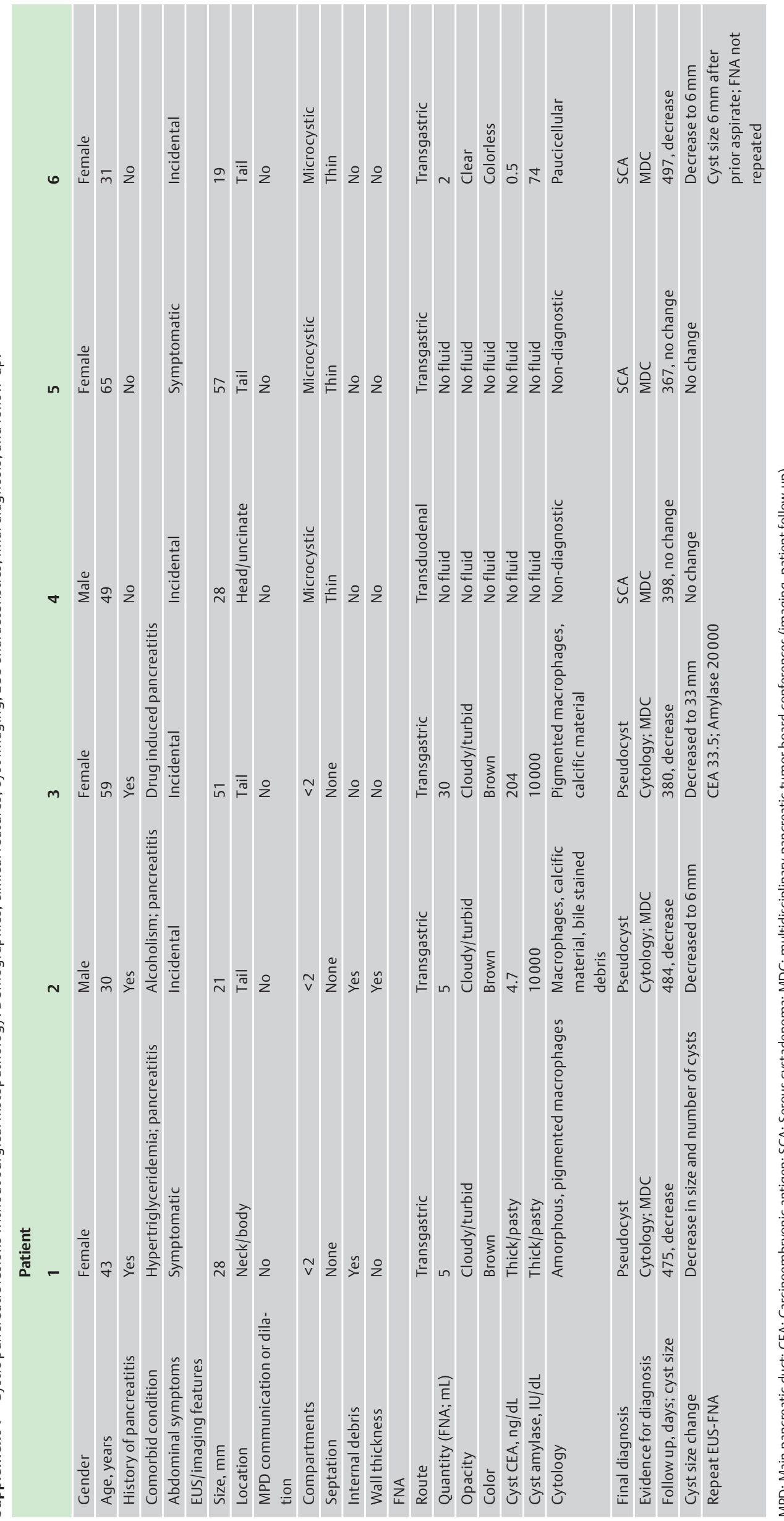


Supplement 2 Paired $t$ test comparison of diagnostic parameters in differentiating mucinous pancreatic cystic lesions using $\mathrm{nCLE}$ patterns between EUS and non-EUS physicians.

\begin{tabular}{|c|c|}
\hline & $P$ value \\
\hline IOA Phase 1 & \\
\hline Sensitivity & 0.218 \\
\hline Specificity & 0.635 \\
\hline Accuracy & 0.856 \\
\hline IOA Phase 2 & \\
\hline Sensitivity & 0.218 \\
\hline Specificity & 0.345 \\
\hline Accuracy & 0.173 \\
\hline
\end{tabular}

EUS, endoscopic ultrasound; nCLE, needle-based confocal laser endomicroscopy. 